\title{
EVALUATION OF DEBRIS EXTRUSION AND SMEAR LAYER PRODUCTION FOLLOWING PREPARATION USING THREE DIFFERENT NICKEL TITANIUM FILES
}

\author{
Reem Adel Abdelgawad" and Bassem Mohamed Eid ${ }^{* *}$
}

\begin{abstract}
Objectives: The aim of this study was to compare the amount of apically extruded debris and the patterns of smear layer formation following the preparation of root canals in extracted human teeth using ProTaper Next, Twisted file, and a reciprocating single-file system.

Material and Methods: Forty-five freshly extracted human single-rooted maxillary incisors were divided into 3 groups $(n=15)$ then instrumented using Protaper Next, Twisted files, and Reciproc. The extruded debris were collected in a pre-weighed Eppendorf tubes. The tubes were stored in an incubator at $70{ }^{\circ} \mathrm{C}$ for 2 days before weighing the dry debris using analytical balance. The debris weight was determined by subtracting the initial weight from the final weight. Root samples were splitted longitudinally into two halves by making grooves then root halves (cervical, middle and apical) thirds were examined using Environmental Scanning Electron Microscope ESEM (FEI Quanta 250 FEG, Berlin, Germany) at $\times 1500$ magnification. All the data were subjected to statistical analysis using Chi-squared, Fisher`s Extract.
\end{abstract}

Results: The three tested rotary nickel titanium files produced comparable amount of debris extrusion and smear layer patterns covering the root canal dentin walls. Apical third showed the heaviest accumulation of smear layer.

KEYWORDS: Debris extrusion, Protaper Next, Twisted file, Reciproc, Smear layer.

\section{INTRODUCTION}

Complete debridement and three-dimensional preparation of the root canal is mandatory for root canal treatment success. However, apical extrusion of debris may lead to postoperative pain ${ }^{1}$, flareups $^{2}$, or development of periapical lesion. Root canal debris is mainly formed of dentin, pulp tissue, microorganisms and their byproducts, root canal filling materials and irrigants. All root canal enlarging preparation techniques could lead to extrusion of debris ${ }^{3,4}$, but the amount of debris may vary according to the design of the instrument or the used technique ${ }^{5}$.

* Assistant Professor of Endodontics, College of Dentistry, Taibah University, Medina, Saudi Arabia, Lecturer of Endodontics, Faculty of Dentistry, Suez Canal University, Ismailia,Egypt

** Assistant Professor of Endodontics, Al-Farabi College of Dentisrty and Nursing, Jeddah, Saudi Arabia, Lecturer of Endodontics, Faculty of Dentistry, Al-Ahram Canadian University, Giza, Egypt 
In 2008, Sybron Endo (Sybron Endo, Orange, CA) presented the first fluted NiTi file manufactured by twisting of the metal, R-phase heat treatment, triangular cross-section, variable pitch, safe-ended tip and special surface conditioning (deoxidation), which significantly increased instrument resistance to cyclic fatigue ${ }^{6}$.

ProTaper Next files (PTN) (Dentsply Maillefer, Ballaigues, Switzerland) have been introduced with an off-centred rectangular cross-section design for greater strength and for producing a mechanical wave of motion that travels along the active length of the file (swaggering effect) as described by the manufacturer. The offset design helps remove debris out of the canal compared with an instrument with a centered mass and axis of rotation ${ }^{7}$.

The Reciproc (VDW, Munich, Germany) singlefile system with a cross-sectional S-shape along the entire length of the working part and sharp cutting edges allows greater cutting efficiency feature a specific motor that performs the reciprocating motion (i.e., movements alternating clockwise and counterclockwise) and made of heat-treated nickeltitanium (Memory Wire, Dentsply Tulsa Dental Specialties, Tulsa, OK, USA), which is resistant to fatigue ${ }^{8}$.

The aim of this in vitro study was to compare the amount of apically extruded debris after the preparation of root canals in extracted human teeth using ProTaper, Twisted file, and a reciprocating single-file system.

\section{MATERIAL AND METHODS}

\section{Teeth Selection and root canals preparation}

Forty-five freshly extracted human single-rooted maxillary incisors were selected for this study. Radiographs were taken to verify single canal. Teeth surfaces were cleaned from soft tissue and calculus using an ultrasonic scaler. Standardizing $16 \mathrm{~mm}$ length root samples by cutting off their crowns then stored in $10 \%$ formalin till use. Canal patency and working length were established using\#10 K-file (Mani Inc, Japan) introduced into each canal till it was just flushed at the apical foramen then subtracting $1 \mathrm{~mm}$ from this measurement. For debris collection; a debris collection apparatus that described by Meyers and Montgomery ${ }^{9}$ was used.

Three consecutive weigh measurements for each empty glass vials without covers were taken with an electronic balance (Precisa; Precisa Inst., Dietikon, Switzerland) with a precision of $10-4 \mathrm{~g}$, and the mean weight was calculated. On the other hand; five control vials containing $2 \mathrm{ml}$ of distilled water were dried and then weighted by the same pre-collection procedures as outlined above to see if the distilled water (used as the irrigant) left any residue.

Root samples were mounted in hole created within the rubber cover of the glass vial, fixed using cyanoacrylate leaving only $1 \mathrm{~mm}$ of the root samples out while the remainder of the root sample suspended in the glass vial. A 25-gauge needle was inserted alongside the stopper to equalize the air pressure inside and outside the vial. All vials were covered with aluminum foil to prevent viewing debris extrusion during the experiment.

Samples were randomly divided into three groups each of $15(\mathrm{~N}=15)$ according to the system used in cleaning and shaping and the files were cycled using VDW Silver Reciproc endodontic motor (VDW, Munich, Germany); Group P where root canals preparation done using ProTaper Next system (Dentsply Maillefer, New York, USA) according to the manufacturer's recommendations at $300 \mathrm{RPM} / 2 \mathrm{Ncm}$ torque in the following sequence: $\mathrm{X} 1$ instrument (taper 0.04, Size 17); X2 instrument (taper 0.06, Size 25); X3 instrument (taper 0.07, Size 30) and then X4 (taper 0.06, Size 40). Group $\mathrm{T}$ where root canals preparation done using Twisted File (TF; SybronEndo, Orange, CA, USA) at a speed of $500 \mathrm{rpm} / 2 \mathrm{Ncm}$ torque as recommended by the manufacturer in the following sequence; $0.08 / 25,0.06 / 30$ and $0.04 / 40$. Group $\mathrm{R}$ where root canals preparation done using Reciproc single file 
system (VDW, Munich, Germany) size 0.06/40 used in a reciprocating slow, in-and out pecking motion at the RECIPROC ALL mode according to the manufacturer instructions. The root canals canal were flooded with $2 \mathrm{ml}$ of distilled water using a 30-gauge side-vented needle (Dentsply Maillefer, Shanghai, China) before instrumentation, between each file size and after completing instrumentation. The needle tip was placed no closer than $3 \mathrm{~mm}$ from the foramen opening in all teeth, and it was never allowed to bind.

Debris weighing: After performing the instrumentation, the aluminum foil cover, needle were removed, and root samples were separated from the glass vial cover, and the debris adhered to the root surface was collected by washing the root with $1 \mathrm{~mL}$ of distilled water in the glass vial. The glass vials were then stored in an incubator at $70{ }^{\circ} \mathrm{C}$ for 2 days to evaporate the distilled water before weighing the dry debris ${ }^{10}$ using the same analytical balance used to weigh the empty glass vials. Three consecutive weights were obtained and averaged and by subtracting the initial weight from this weight the extruded debris was determined.

Root canal wall cleanliness: Root samples were splitted longitudinally into two halves by making grooves on the external buccal and lingual surfaces using a diamond disk and split carefully with the use of a fine osteotome. An absorbent paper was left inside the root canals to prevent the dentin dust coming from the external cut, from penetrating into root canal walls. Root halves (cervical, middle and apical) thirds were examined using Environmental Scanning Electron Microscope ESEM (FEI Quanta 250 FEG, Berlin, Germany) at $\times 1500$ magnification . Digital images were recorded then; root canal wall cleanliness evaluated using Image J program (U.S. National Institutes of Health, Bethesda, Maryland, USA) according to a three-score system developed by Zmener scoring ${ }^{11}$ where pulp remnants, dentine chips, larger particles and aggregates appearing haphazardly on the root canal walls were classified as debris. A score 1 was assigned when no debris or isolated small particles were present. Score 2 indicated that debris covered more than $50 \%$ of the canal walls and a score 3 indicated that debris almost entirely covered the canal walls.

\section{RESULTS}

Mean and standard deviation were estimated from the sample for each Study group. The mean values were compared by one-way ANOVA followed by post hoc tukey test to identify the significant groups (table 1). The significance level was set at $\mathrm{P} \leq 0.05$. Statistical analysis was performed with IBM ${ }^{\circledR}$ SPSS ${ }^{\circledR}$ Statistics Version 20 for Windows. There was no statistically significant difference in debris extrusion between the three tested groups. Least amount of debris extrusion was observed with Protaper Next Rotary Ni-Ti (group P) while, Reciproc Ni-Ti (group R) Instruments extruded highest debris amongst test groups (Fig 1).

TABLE (1) Amount of apically extruded debris after the use of the different file systems.

\begin{tabular}{|c|c|c|c|c|c|}
\hline Group & $\mathbf{N}$ & Mean & SD & Min & Max \\
\hline $\mathbf{P}$ & $\mathbf{1 5}$ & 0.002894 & 0.000318 & 0.00234 & 0.00357 \\
\hline $\mathbf{T}$ & $\mathbf{1 5}$ & 0.002977 & 0.000228 & 0.00248 & 0.00335 \\
\hline $\mathbf{R}$ & $\mathbf{1 5}$ & 0.003075 & 0.000281 & 0.00264 & 0.00381 \\
\hline
\end{tabular}

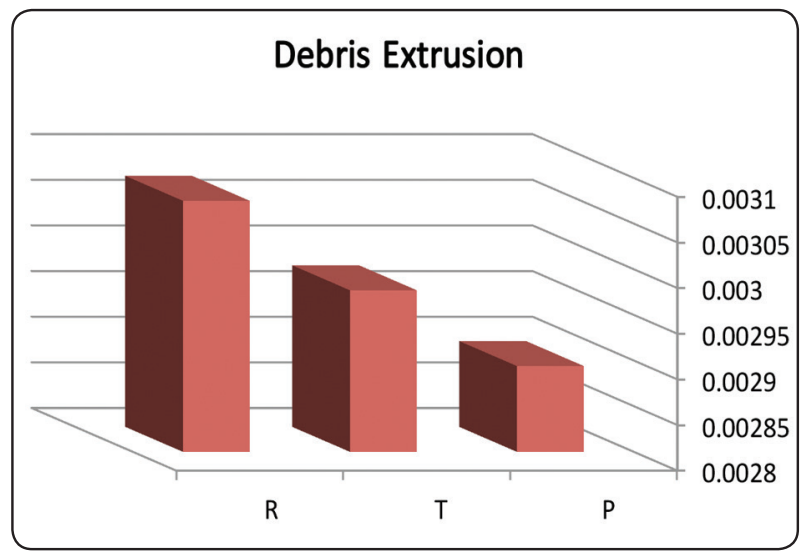

Fig. (1) The mean counts, of extruded debris between experimental groups. 


\section{Root canal wall cleanliness}

Qualitative variables were demonstrated in the form of number and frequency. Chi-squared $\left(\mathrm{x}^{2}\right)$, Fisher's Extract (FE) test were used to figure out if there were statistically significant difference in between groups. A p-value of less than 0.05 was considered significant.
As shown in the table (2) all the groups showed similar amounts of smear layer at the coronal and middle thirds while at the apical thirds smear layer mean scores was statistically significant.

Also, there was statistically significant difference between scores regarding files used in the apical third.

TABLE (2) Mean debris scores $( \pm \mathrm{SD})$ recorded in the apical, middle, and coronal thirds of canals prepared by PTN, Reciproc, TF rotary instruments.

\begin{tabular}{|c|c|c|c|c|}
\hline Third & File & Mean \pm SD & p-value across thirds & p-value across files \\
\hline \multirow{3}{*}{ Coronal } & Protaper Next & $2.53 \pm 0.52$ & \multirow{3}{*}{0.365} & \multirow{9}{*}{$\underline{0.002}$} \\
\hline & Reciproc & $2.53 \pm 0.52$ & & \\
\hline & Twisted File & $2.47 \pm 0.52$ & & \\
\hline \multirow{3}{*}{ Middle } & Protaper Next & $2.67 \pm 0.49$ & \multirow{3}{*}{0.637} & \\
\hline & Reciproc & $2.6 \pm 0.51$ & & \\
\hline & Twisted File & $2.4 \pm 0.74$ & & \\
\hline \multirow{3}{*}{ Apical } & Protaper Next & $2.8 \pm 0.41$ & \multirow{3}{*}{$\underline{0.004}$} & \\
\hline & Reciproc & $2.73 \pm 0.46$ & & \\
\hline & Twisted File & $3 \pm 0$ & & \\
\hline
\end{tabular}

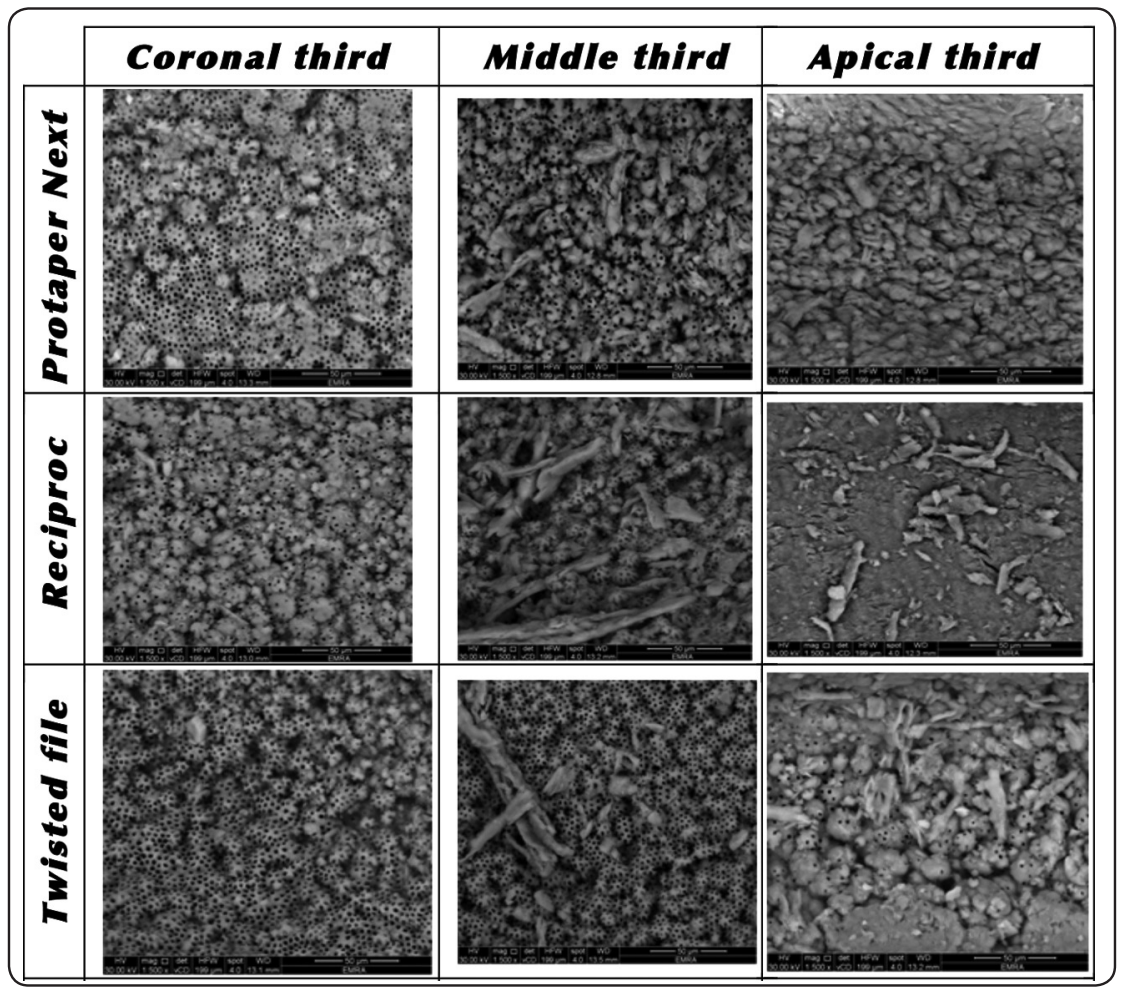

Fig. (2) SEM photomicrograph at x 1500 magnification showing smear layer formation following preparation by the three tested rotary files at coronal, middle and apica 


\section{DISCUSSION}

\section{Debris extrusion}

During root canal treatment some complications as, postoperative pain and interappointment flare ups is a consequences of debris extusion ${ }^{1}$. However, there is no technique till now has been proved to avoid apical extrusion of debris ${ }^{3,12}$.

The design of the instrument, mode of action, irrigant used, and curvature of the root could affect the quantity of the debris extruded from the apical foramen during preparation ${ }^{13,14}$. In this study we have tested three rotary nickel titanium systems with different designs, manufacturing methods, number of files and technique of cutting.

Protaper Next files are made of M-Wire to increase flexibility and to improve cyclic fatigue, has off-centred rectangular cross-section apical portion design, which provides the nonuniform and reduced contact points between the instrument and the root canal wall which could lead to the removal of more debris in the coronal direction and result in less debris extrusion ${ }^{15}$.

TF can work at speeds up to $1500 \mathrm{rpm}$, the manufacturer suggests that using a speed of 500 rpm which is utilized in this study is likely to reduce waste and increase the service life of the instrument. Underlining that using higher rotational speed will lead to less debris extrusion ${ }^{16}$.

In our study to collect the debris we have used the model used by Myers and Montgomery ${ }^{9}$ which is widely accepted although it lacks the presence of material as agar to simulate the presence periodontal tissues apical pressure which can resist the extrusion of debris in vivo. Sodium hypochlorite was replaced as a root canal irrigant by distilled water to avoid crystallization which could possibly affect our results $^{17}$.

Our results have showed that the three tested files produced apical extrusion of debris but there was no statistically significant difference between the three tested instruments. This comes in accordance with results of Üstün et al ${ }^{18}$ who found there was no statistically significant difference between PTN and $\mathrm{TF}$, also Capar et al ${ }^{19}$ found there was no statistically significant difference between PTN and TF adaptive (TFA) which have the same design and metallurgy of the TF with different kinetics as it changes its motion from full rotation to reciprocation when subjected to stresses. On the contrary Dincer et $\mathbf{a l}^{20}$ found that PTN produced more apical debris than TFA Regarding the results of the Reciproc file our results showed no statistically significant difference between the Reciproc, PTN and TF; meanwhile the review have shown contradictory regarding the reciprocating movement in general versus the full rotation motion as Kocak ${ }^{21}$ et al found that Reciproc file produced less apical debris when compared with other systems as Revo-s SU and ProTaper F2 and the same results were reported by Tinoco et al $^{22}$, Üstün et $\mathbf{a l}^{18}$ when they compared single file reciprocating system to multifiles full rotation systems. On the contrary Burklein ${ }^{23}$ et al reported that single file reciprocating systems produce more apical debris than full rotation files and he explained his results by the higher cutting efficiency of Reciproc due to S-shaped cross section in comparison of the triangular cross section of ProTaper and WaveOne and therefore more debris were produced and pushed through the apical foramen, but in our study the difference in cross section has no impact on our results as there was no statistically significant difference between the three systems.

Absence of back pressure from the periapical tissues may have caused the irrigant by the force of gravity to carry the debris out of the apical foramen in all tested systems that's why the use of foam to simulate the resistance of periapical tissues was suggested $^{24,25}$.

In conclusion; under the condition of this study, the null hypothesis in our study was accepted as Reciprocation motion has no advantage over the full rotation motion in preventing extrusion of apical debris. 


\section{Root canal cleanliness}

The primary objective of root canal instrumentation is to three-dimensional shaping of the root canal and to clean the root canal system. Smear layer production is considered unavoidable during instrumentation. It has an average film thickness $1-2 \mu$ covering the root canal dentin walls which can prevent effective removal of microorganisms and sealing of the root canal system $^{26}$.

For decades SEM has been used for many years to give high-resolution evaluation of smear layer covering dentin surface. The advantage of using ESEM in the present study is operating in wet mode as it is not necessary to make nonconductive samples conductive as the samples do not need to be desiccated and coated with gold or palladium and thus their original characteristics may be preserved for further testing or manipulation ${ }^{27}$.

While considerable effort has been directed toward ways to remove the smear layer fewer studies showed interest on the formation of smear layer at different levels. The interest of our study was to evaluate the difference in smear layer formation using different root canal files at the three anatomical levels; so, the irrigant used was $2 \mathrm{ml}$ of distilled water and there was no use either for $\mathrm{NaOCl}$ or EDTA to remove the organic and inorganic part of smear layer.

Using the scoring system used by Zmener ${ }^{11}$ et al described earlier; the results in our study showed that smear layer formation in the apical third was higher than coronal and middle, which can be explained by inability of the irrigant to penetrate the apical third which came along with Peters ${ }^{28}$ et al who found no difference in cleanliness of root canal when water was used as a root canal irrigant and also with Narayan ${ }^{29}$, Zarei $^{30}$ et al who found that the preparation in the coronal and middle thirds were cleaner than the apical regardless of the instrument used.

The increase in smear layer in the apical third may be due to the inability of distilled water to reach the apical third due to depth of penetration of the needle or the size of apical enlargement which was proved by Khademi ${ }^{31}$ et al to be a decisive factor in cleanliness of the root canal in apical third.

Regarding the difference between the root canal files used in preparing the root canal there was no statistically significant difference between the files at the coronal and middle thirds while at the apical Twisted file showed the highest in smear layer production, this come in accordance with Grecca $^{32}$ et al who proved that neither instruments nor instrumentation techniques are capable of complete cleanliness of the root canal system.

\section{CONCLUSIONS}

- $\quad$ The three tested rotary nickel titanium files produced comparable amount of debris extrusion and smear layer patterns covering the root canal dentin walls.

- Thickness of the smear layer was greater in apical third regardless of the used file.

- ESEM is an excellent tool when considering studying the ultra-structural morphology of dentin.

\section{REFERENCES}

1. Siqueira JF Jr, Rocas IN, Favieri A, et al. Incidence of postoperative pain after intracanal procedures based on an antimicrobial strategy. J Endod 2002; 28:457-60.

2. Seltzer S, Naidor f IJ. Flare-ups in endodontics: I. Etiological factors. J Endod 1985; 11:472-8.

3. Al-Omari MA, Dummer PM Canal blockage and debris extrusion with eight preparation techniques. J Endod 1995 , 21:154-158.

4. Ferraz CC, Gomes NV, Gomes BP Apical extrusion of debris and irrigants using two hand and three engine-driven instrumentation techniques. Int Endod J 2001; 34: 354-8.

5. Kustarcı A, Akpınar KE. Comparative study of apically extruded debris using one manual and two rotary instrumentation techniques for endodontic retreatment J Dent Sci 2012; 7:1-6. 
6. Gambarini G, Grande NM, Plotino G. Fatigue resistance of engine-driven rotary nickel-titanium instruments produced by new manufacturing methods. J Endod 2008; 34:1003-5.

7. Ruddle CJ, Machtou P, West JD. 2013. The shaping movement: fifth-generation technology, Dent Today; 32(4):96-99.

8. Yared G, Ramli AG. Single file reciprocation: A literature review. ENDO (lond Engl) 2013;7(3):171-8.

9. Myers GL, Montgomery S. A comparison of weights of debris extruded apically by conventional filing and Canal Master techniques. J Endod 1991; 17:275-9.

10. Singh A, runagiri D, Pushpa S, Sawhny A, Misra A, Khetan K. Apical extrusion of debris and irrigants using ProTaper hand, M-two rotary and WaveOne single file reciprocating system: An ex vivo study cons D.2015; 18 (5): 405-8.

11. Zmener O, Pameijer $\mathrm{CH}$, Banegas G. Effectiveness in cleaning oval-shaped root canals using Anatomic Endodontic Technology, ProFile and manual instrumentation: a scanning electron microscopic study. International Endodontic Journal 2005, 38, 356-363.

12. Hinrichs RE, Walker WA, Schindler WG. A comparison of amounts of apically extruded debris using handpiecedriven nickel titanium instrument systems. J Endod 1998; 24:102-106.

13. Ferraz C, Gomes N, Gomes B, Zaia A, Teixeira F, SouzaFilho F. Apical extrusion of debris and irrigants using two hand and three engine-driven instrumentation techniques. International Endodontic Journal 2001;34, 354-8.

14. Tinaz AC, Alacam T, Uzun O, Maden M, Kayaoglu G. The effect of disruption of apical constriction on periapical extrusion. Journal of Endodontics 2005; 31, 533-5.

15. El Naghy AM Cyclic fatigue resistance of ProTaper Next nickel-titanium rotary files. International Endodontic Journal. 2014; 6,1034-9.

16. Liu M, Xiong S, Tan F, Liu Y. Less extrusion debris during the retreatment of curved canals using twisted files with higher rotational speeds: an ex vivo study. BMC Oral Health 2017; 17:45.

17. Tanalp J, Gungor T. Apical extrusion of debris: a literature review of an inherent occurrence during root canal treatment. International Endodontic Journal 2014; 47, 211-21.

18. Ustun Y, Canakci BC, Dincer AN, Er O, Duzgun S Evaluation of apically extruded debris associated with several Ni-Ti systems. International Endodontic Journal 2015; 48,701-4.

19. Capar ID, Arslan H, Akcay M, Ertas H. An in vitro comparison of apically extruded debris and instrumentation times with ProTaper Universal, ProTaper Next, Twisted File Adaptive, and HyFlex instruments. Journal of Endodontics 2014; 40, 1638-41.

20. Dincer AN, Guneser MB, and Arslan D. Apical extrusion of debris during root canal preparation using a novel nickel-titanium file system: Wave One gold. J consevr Dent 2017; 20 (5):322-325.

21. Koçak MM, Çiçek E, Koçak S, Sağlam BC, Turker SA. Apical Extrusion of Debris Using Self-Adjusting File, Reciprocating Single-file, and 2 Rotary Instrumentation Systems. J Endod 2013; 39(10):1278-80.

22. Tinoco J,De-Deus G, Tinoco E, SaavedraF, FidelR, Sassone L. Apical extrusion of bacteria when using reciprocating single-file and rotary multifile instrumentation systems. International Endodontic Journal 2013; 47, 560-6.

23. Bürklein S, Schäfer E. Apically extruded debris with reciprocating single-file and full sequence rotary instrumentation systems. Journal of Endodontics 2012; 38, 850-2.

24. Hachmeister DR, Schindler WG, Walker WA, et al. The sealing ability and retention characteristics of mineral trioxide aggregate in a model of apexification. J Endod 2002; 28:386-90.

25. Altundasar E, Nagas E, Uyanik O, et al. Debris and irrigant extrusion potential of 2 rotary systems and irrigation needles. Oral Surg Oral Med Oral Pathol Oral Radiol Endod 2011; 112:31-5.

26. American Association of Endodontists. Glossary Contemporary Terminology for Endodontics, 6th Edition. American Association of Endodontists, Chicago III, USA. 1998.

27. McDonald AM. Environmental scanning electron microscopy. Mater World 1998; 6:399-401.

28. Peters OA, Barbakow F (2000) Effects of irrigation on debris and smear layer on canal walls prepared by two rotary techniques: a scanning electron microscopic study. Journal of Endodontics 26, 6-10.

29. Narayan GS, Venkatesan SM, Karumaran CS, Indira R, Ramachandran S, Srinivasan MR. A comparative evaluation on the cleaning and shaping ability of three nickel titanium rotary instruments using computerized tomography - An ex vivo study. Cont Clin Dent. 2012; 3:151-155. 
30. Zarei M, Javidi M, Afkhami F, Tanbakuchi B, Zadeh MM, Mohammadi MM. Influence of Root Canal Tapering on Smear Layer Removal. The New York State Dental Journal 2016, April 35-8.

31. Khademi A, Yazdizadeh M, Feizianfard M. Determination of the minimum instrumentation size for penetration of irrigants to the apical third of root canal systems. J Endod 2006; 32:417-20.

32. Grecca FS, Garcia RB, Bramante CM, Moraes IG, Bernardineli N. A quantitative analysis of rotary, ultrasonic and manual techniques to treat proximally flattened root canals. J Appl Oral Sci. 2007; 15:89-93. 